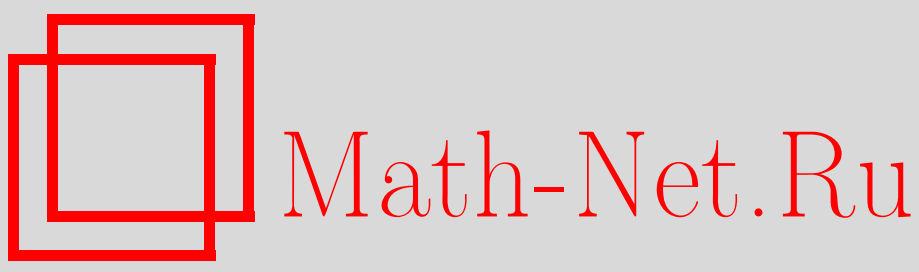

А. А. Милютин, Об условиях неотрицательности интегральных квадратичных форм с постоянными коэффициентами, определенных на полуоси, Матем. сб., 2002, том 193, номер 4, 61-86

DOI: https://doi.org/10.4213/sm644

Использование Общероссийского математического портала Math-Net.Ru подразумевает, что вы прочитали и согласны с пользовательским соглашением http://www.mathnet.ru/rus/agreement

Параметры загрузки:

IP : 54.162 .27 .143

26 апреля 2023 г., 13:28:36 
А.А. Милютин

\section{Об условиях неотрицательности интегральных квадратичных форм с постоянными коэффициентами, определенных на полуоси}

Рассматривается интегральный квадратичный функционал с постоянными коэффициентами, определенный на полуоси. Дается необходимое и достаточное условие его неотрицательности на всех парах функций, связанных линейньм дифоференциальньм уравнением и интегрируемых с квадратом, основанное на неравенстве Гамильтона-Якоби. Установлена связь полученного условия с известным частотньм критерием.

Библиографоя: 3 названия.

\section{§1. Постановка вопроса}

Пусть $x \in \mathbb{R}^{d(x)}, u \in \mathbb{R}^{d(u)}, d(u) \leqslant d(x)$ (через $d(a)$ обозначим размерность вектора $a$ ). Через $L$ обозначим линейное многообразие финитных пар $(x(t), u(t)) \mid$ $t \in[0, \infty)$, т.е. таких, которые отличны от нуля лишш на ограниченном множестве. Функцию $x(\cdot)$ мы будем считать абсолютно непрерывной и равной нулю при $t=0$, функцию $u(t)$ - измеримой и ограниченной на $[0, \infty)$.

Рассмотрим управляемую систему $V: \dot{x}=K x+B u$, где $K$ и $B$-постоянные матрицы. Через $L(V)$ обозначим множество таких элементов $L$, которые являются траекториями $V$. Положим

$$
\Phi(x, u)=u^{2}+2 A x u+Q x x,
$$

где

$$
A: \mathbb{R}^{d(x)} \rightarrow \mathbb{R}^{d(u)}, \quad Q: \mathbb{R}^{d(x)} \rightarrow \mathbb{R}^{d(x)}
$$

- постоянные матрицы, причем $Q$ предполагается симметрической.

Пусть пара $(x(\cdot), u(\cdot)) \in L$. Положим

$$
J(x(\cdot), u(\cdot))=\int_{0}^{\infty} \Phi(x(t), u(t)) d t .
$$

Нас будет интересовать вопрос о необходимых и достаточных условиях выполнения неравенства

$$
J(x, u) \geqslant 0 \quad \forall(x, u) \in L(V) .
$$

Условие, которое мы получим, формально является более слабым, а значит, и более "продвинутым", нежели известное условие, полученное путем разложения по Фурье [1]. 


\section{§2. Формулировка основной теоремы}

Пусть матрицы $A, Q, K, B$ заданы. Через $S$ будем обозначать произвольную симметрическую матрицу. Пусть $S$ задана. Для любой траектории системы $V$ положим:

$$
\Phi_{S}(x(\cdot), u(\cdot))=\Phi(x(\cdot), u(\cdot))+\frac{d}{d t}(S x(t), x(t)) .
$$

Нетрудно видеть, что

$$
\Phi_{S}(x, u)=\left(u+U_{S} x\right)^{2}+\omega_{S}(x),
$$

где

$$
\begin{gathered}
U_{S}: \mathbb{R}^{d(x)} \rightarrow \mathbb{R}^{d(u)}, \quad U_{S}=-\left(A+B^{*} S\right), \\
\omega_{S}(x)=-\left(U_{S} x\right)^{2}+2(K x, S x)+(Q x, x) .
\end{gathered}
$$

Положим для $(x(t), u(t)) \in L(V)$

$$
J_{S}(x(\cdot), u(\cdot))=\int_{0}^{\infty} \Phi_{S}(x(t), u(t)) d t .
$$

Очевидно, что

$$
J_{S}(x(\cdot), u(\cdot))=J(x(\cdot), u(\cdot)) \quad \forall(x, u) \in L(V) .
$$

Пусть $\lambda(S)$ - наименьшее собственное число формы $\omega_{S}(x)$. Обозначим

$$
\widehat{\lambda}=\sup _{S} \lambda(S) .
$$

Основньм результатом настоящей работы является

TеОРема 1. Неравенство $\widehat{\lambda} \geqslant 0$ әквивалентно неравенству $J \geqslant 0$ на $L(V)$.

Выражение для $\omega_{S}(x)$ является левой частью уравнения Гамильтона-Якоби для функции вида $(S x, x)$ при $S=$ const.

Условие $\omega_{S}(x) \geqslant 0$ представляет, таким образом, неравенство ГамильтонаЯкоби. Достаточность условия теоремы очевидна. Главное в ней - необходимость. Мы проведем доказательство, не опираясь на частотный критерий [1].

В случае двумерного пространства и управляемой системы вида $\dot{x}=u$ А.В. Дмитрук [2] исследовал вопрос о неотрицательности $J$ на $L(V)$, опираясь, по существу, на теорему 1 , хотя и не формулируя ее. Он получил новое условие, эквивалентное неотрицательности $J$ на $L(V)$. Оно не связано с частотным критерием. Приведем его формулировку.

Мы будем считать (что допустимо) матрицу $A$ кососимметрической. Пусть $q_{0}, q_{1}$ - собственные числа матрицы $Q$ и $a$ - модуль собственного числа матрицы $A$. Тогда указанное условие состоит в том, чтобы выполнялись неравенства:

$$
q_{0} \geqslant 0, \quad q_{1} \geqslant 0, \quad \sqrt{q_{0}}+\sqrt{q_{1}} \geqslant 2 a .
$$

В следующем параграфе мы приведем леммы, необходимые для доказательства теоремы 1. 


\section{§3. Некоторые леммы}

3.1. Пусть $R: \mathbb{R}^{d(x)} \rightarrow \mathbb{R}^{d(x)}$ - линейный оператор. Имеет место

Лемма 1. Следующие два условия әквивалентны:

(*) $\max _{|x|=1}(R x, \bar{S} x) \geqslant 0$ для любой симметрической матрицы $\bar{S}$;

(**) существует собственное число матрищъ $R$ с нулевой вещественной частью.

ДокАЗАТЕЛьство. Сначала мы докажем, что

$$
(* *) \Rightarrow(*) .
$$

Действительно, если $\lambda=0$ - собственное число матрищы $R$, то существует вектор $x_{*}$ такой, что $R x_{*}=0,\left|x_{*}\right|=1$. Следовательно, для любой матрицы $\bar{S}$ выполнено равенство $\left(R x_{*}, \bar{S} x_{*}\right)=0$ и, тем самым, выполнено $(*)$.

Пусть теперь $\lambda=i \sigma, \sigma \neq 0,-$ собственное число матрицы $R$. Тогда, как известно, сушествует решение уравнения $\dot{x}=R x$ вида:

$$
x^{0}(t)=x_{0} \cos \sigma t+x_{1} \sin \sigma t
$$

где векторы $x_{0}$ и $x_{1}$ линейно независимы.

Пусть $\bar{S}$ - произвольная симметрическая матрица. Рассмотрим функцию $\frac{1}{2} \bar{S} x^{0}(t) x^{0}(t)$. Пусть $t_{*}-$ точка максимума этой функции. Тогда ее производная в $t_{*}$ обращается в нуль. Следовательно, $\left(R x^{0}\left(t_{*}\right), \bar{S} x^{0}\left(t_{*}\right)\right)=0$. Но $x^{0}\left(t_{*}\right) \neq 0$, ибо $x_{0}$ и $x_{1}$ линейно независимы. Тем самым для $R$ выполнено $(*)$.

Итак, (3.1) доказано. Теперь мы докажем, что если условие (**) не имеет места, то и условие $(*)$ также не имеет места.

Действительно, исходя из жордановой формы матрицы $R$ можно утверждать, что пространство $\mathbb{R}^{d(x)}$ разлагается в прямую сумму подпространств, инвариантных относительно $R$, в каждом из которых $R$ можно представить в одном из двух видов.

1) Пусть $X$ - одно из таких слагаемых. Тогда в некотором базисе в $X$ оператор $R$ имеет вид:

$$
\begin{aligned}
& y_{1}=\lambda x_{1}+x_{2}, \\
& y_{k}=\lambda x_{k}+x_{k+1}, \quad k \leqslant n-1, \\
& y_{n}=\lambda x_{n}
\end{aligned}
$$

где $\lambda$-вещественное число, $n=d(X)$.

2) Пусть $d(X)=2 m$. Нам удобно представить элемент подпространства $X$ в виде: $\left(\xi_{1}, \eta_{1}, \xi_{2}, \eta_{2}, \ldots, \xi_{m}, \eta_{m}\right)$, т.е. все нечетные компоненты обозначить через $\xi$, а все четные - через $\eta$. Существует базис, в котором оператор $R$ имеет вид:

$$
\begin{aligned}
\bar{w}_{1} & =\lambda w_{1}+\sigma \bar{V} w_{1}+w_{2}, \\
\bar{w}_{k} & =\lambda w_{k}+\sigma \bar{V} w_{k}+w_{k+1}, \quad k \leqslant m-1, \\
\bar{w}_{m} & =\lambda w_{m}+\sigma \bar{V} w_{m}
\end{aligned}
$$


где

$$
w_{k}=\left(\xi_{k}, \eta_{k}\right), \quad \bar{V} w=(\bar{\xi}, \bar{\eta}), \quad \bar{\xi}=-\eta, \quad \bar{\eta}=\xi, \quad \sigma \neq 0 .
$$

В первом случае $\lambda$ - вешественное собственное число оператора $R$, во втором случае пара $\lambda \pm i \sigma$ - сопряженные собственные числа $R$. Таким образом, $\lambda \neq 0$ во всех случаях. В дальнейшем мы будем говорить о слагаемых первого и второго рода в зависимости от того, является собственное число вешественным или нет. В каждом слагаемом определим квадратичную форму следуюшим образом.

Пусть $X$ - слагаемое первого рода. В базисе, в котором $R$ представлен в виде (3.2), положим

$$
Z_{X}(x)=\sum_{k=1}^{d(X)} s_{k} x_{k}^{2}
$$

Коэффициенты $s_{k}$ выберем следующим образом. Имеем:

$$
\frac{1}{2} \frac{d}{d t} Z_{X}(x)=\sum_{k=1}^{d(X)} \lambda s_{k} x_{k}^{2}+\sum_{k=1}^{d(X)-1} s_{k} x_{k} x_{k+1}
$$

где $\frac{d x}{d t}=R x$. Потребуем, чтобы $\operatorname{sign} s_{k}=-\operatorname{sign} \lambda \forall k$. Далее потребуем, чтобы $\left|s_{k}\right|$ столь быстро вырастал с ростом $k$, что для любого $x \neq 0$ имело бы место неравенство

$$
\frac{1}{2} \frac{d}{d t} Z_{X}(x)<0
$$

Нетрудно видеть, что эти требования выполнимы.

Для слагаемого второго рода положим

$$
Z_{X}(w)=\sum_{k=1}^{\frac{1}{2} d(X)} s_{k} w_{k}^{2}, \quad \text { где } w_{k}^{2}=\xi_{k}^{2}+\eta_{k}^{2}
$$

Тогда

$$
\frac{1}{2} \frac{d}{d t} Z_{X}(w)=\sum_{k=1}^{\frac{1}{2} d(X)} \lambda s_{k} w_{k}^{2}+\sum_{k=1}^{\frac{1}{2} d(X)-1} s_{k} w_{k} w_{k+1}, \quad \frac{d w}{d t}=R w
$$

Мы также потребуем, чтобы $\operatorname{sign} s_{k}=-\operatorname{sign} \lambda$ и чтобы рост $\left|s_{k}\right|$ обеспечивал неравенство:

$$
\frac{1}{2} \frac{d}{d t} Z_{X}(w)<0 \quad \forall w \neq 0 .
$$

Так же как и выше, нетрудно заметить, что это требование выполнимо.

Итак, формы $Z_{X}$ выбраны. Положим $\widehat{Z}(x)=\sum_{X} Z_{X}(x)$. В исходном базисе имеем $\widehat{Z}(x)=(\widehat{S} x, x)$, где $\widehat{S}$ - симметрическая матрица. По построению $\frac{1}{2} \frac{d}{d t} \widehat{Z}(x)<0 \forall x \neq 0$. Следовательно, $(R x, \widehat{S} x)<0 \forall x \neq 0$. Отсюда видно, что условие $(*)$ не имеет места. Лемма доказана. 
3.2. Пусть $\Gamma$ - ненулевое подпространство в $\mathbb{R}^{d(x)}$. Рассмотрим условие:

$(* ; \Gamma) \max \{(R x, S x): x \in \Gamma,|x|=1\} \geqslant 0$ для любой симметрической матрицы $S$.

Лемма 2. Пусть условие $(* ; \Gamma)$ выполнено. Тогда существует подпространство $\widehat{\Gamma}$ такое, что

$$
\widehat{\Gamma} \subset \Gamma, \quad d(\widehat{\Gamma})>0, \quad R \widehat{\Gamma} \subset \widehat{\Gamma}
$$

и условие $(* ; \widehat{\Gamma})$ выполнено.

ДокАЗАТЕЛЬСТво. Для произвольного оператора $M: \mathbb{R}^{d(x)} \rightarrow \mathbb{R}^{d(x)}$ положим

$$
\begin{aligned}
& M_{1}: \Gamma \rightarrow \mathbb{R}^{d(x)}, \quad M_{1} x=P_{\Gamma} M x, \\
& M_{2}: \Gamma \rightarrow \mathbb{R}^{d(x)}, \quad M_{2} x=P_{\Gamma^{\prime}} M x,
\end{aligned}
$$

где $P_{\Gamma}$ и $P_{\Gamma^{\prime}}$ - ортогональные проекторы на Г и его ортогональное дополнение $\Gamma^{\prime}$.

Заметим, что оператор $M$ совпадает на $\Gamma$ с некоторьм симметрическим оператором $S$ тогда и только тогда, когда $M_{1}$ - симметрический оператор на $Г$. Что касается $M_{2}$, то он произволен. Справедливость данного утверждения проверяется элементарно.

Теперь мы можем перейти непосредственно к доказательству леммы.

Если $R_{2}=0$, то лемма верна, ибо в этом случае $\widehat{\Gamma}=\Gamma$. Допустим, что $R_{2} \neq 0$. Докажем, что тогда $d\left(R_{2} \Gamma\right)<d(\Gamma)$. Действительно, в противном случае, выбирая $S$ из условий $S_{1}=0, S_{2}=-R_{2}$, получаем:

$$
\left.(S x, R x)\right|_{\Gamma}=-\left(R_{2} x\right)^{2} .
$$

Но поскольку $d\left(R_{2} \Gamma\right)=d(\Gamma)$, то $(S x, R x)<0 \forall x \in \Gamma, x \neq 0$. Однако это противоречит условию леммы.

Итак, $d\left(R_{2} \Gamma\right)<d(\Gamma)$. Положим $\Gamma_{1}=R_{2}^{-1}(0)$. Очевидно, $0<d\left(\Gamma_{1}\right)<d(\Gamma)$. Докажем, что для $R$ имеет место условие $\left(* ; \Gamma_{1}\right)$.

Допустим противное - пусть существует симметрическая матрица $S$ такая, что $(S x, R x)<0 \forall x \in \Gamma_{1}, x \neq 0$. Выберем $\widehat{S}$ из условий $\widehat{S}_{1}=S_{1}, \widehat{S}_{2}=-N R_{2}$, где $N$ - некоторое вешественное число. Тогда $\left.(\widehat{S} x, R x)\right|_{\Gamma}=\left(S_{1} x, R_{1} x\right)-N\left(R_{2} x\right)^{2}$. Положим:

$$
x=x^{\prime}+x^{\prime \prime}, \quad x^{\prime} \in \Gamma_{1}, \quad x^{\prime \prime} \perp \Gamma_{1}, \quad x, x^{\prime \prime} \in \Gamma .
$$

Тогда

$\left.(\widehat{S} x, R x)\right|_{\Gamma}=\left(S_{1} x^{\prime}, R_{1} x^{\prime}\right)+\left(S_{1} x^{\prime}, R_{1} x^{\prime \prime}\right)+\left(S_{1} x^{\prime \prime}, R_{1} x^{\prime}\right)+\left(S_{1} x^{\prime \prime}, R_{1} x^{\prime \prime}\right)-N\left(R_{2} x\right)^{2}$.

Но по определению $\Gamma_{1}$ имеем $R_{2} x^{\prime}=0$. Следовательно, последнее слагаемое можно записать в виде $-N\left(R_{2} x^{\prime \prime}\right)^{2}$. По этой же причине $\left(S_{1} x^{\prime}, R_{1} x^{\prime}\right)=\left(S x^{\prime}, R x^{\prime}\right)$ и, следовательно, эта величина не превосходит $-\alpha_{0} x^{\prime 2}$, где $\alpha_{0}>0$. Далее, так как $\left(R_{2} x^{\prime \prime}\right)^{2} \geqslant \alpha_{1} x^{\prime \prime 2}$, где $\alpha_{1}>0$, то, выбирая $N$ достаточно большим, получаем:

$$
(\widehat{S} x, R x)<0 \quad \forall x \in \Gamma, x \neq 0,
$$

что противоречит условию $(* ; \Gamma)$. Итак, мы доказали, что если Г не инвариантно относительно $R$, то существует $\Gamma_{1}$ такое, что $0<d\left(\Gamma_{1}\right)<d(\Gamma)$ и выполнено условие $\left(* ; \Gamma_{1}\right)$. Отсюда легко следует утверждение леммы. 
3.3. Пусть $\omega(x, y)=C x x+D x y+E y y-$ квадратичная форма в $\mathbb{R}^{2 d(x)}$. Мы предположим, что $E$ - неотрицательно определенная матрица. Пусть $S$ - симметрическая матрища, $\Gamma$ - подпространство в $\mathbb{R}^{d(x)}$. Положим

$$
f(S, \Gamma)=\max _{x \in \Sigma(\Gamma)} \omega(x, S x)
$$

где

$$
\Sigma(\Gamma)=\{x \in \Gamma:|x|=1\} \text { - единичная сфера в } \Gamma .
$$

Если $\Gamma=\{0\}$, то условимся считать $f(S, \Gamma)=-\infty \forall S$.

Положим $f(\Gamma)=\inf _{S} f(S, \Gamma)$. Пространство симметрических матриц обозначим через $\mathscr{J}$. Скалярное произведение в $\mathscr{J}$ определим как сумму произведений одноименных элементов.

Положим

$$
\mathscr{J}_{0}(\Gamma)=\{S:(D x, S x)=0,(E S x, S x)=0 \text { на } \Gamma\} .
$$

Так как $E \geqslant 0$, то второе условие эквивалентно условию $E S=0$ на Г. Следовательно, $\mathscr{J}_{0}(\Gamma)$ - подпространство в $\mathscr{J}$ для любого $Г$. Через $\mathscr{J}_{1}(\Gamma)$ обозначим ортогональное дополнение к $\mathscr{J}_{0}(\Gamma)$ в $\mathscr{J}$.

Введем два определения:

$$
\begin{aligned}
& \Omega(\Gamma)=\left\{S \in \mathscr{J}_{1}(\Gamma):(D x, S x) \leqslant 0,(E S x, S x)=0 \text { на } \Gamma\right\}, \\
& \widehat{\Gamma}(\Gamma)=\{x \in \Gamma:(D x, S x)=0 \forall S \in \Omega(\Gamma)\} .
\end{aligned}
$$

Очевидно, что $\Omega(\Gamma)$ - выпуклый конус в $\mathscr{J}_{1}(\Gamma)$. Так как

$$
S \in \Omega(\Gamma) \Rightarrow(D x, S x) \leqslant 0 \text { на } \Gamma,
$$

то условие $(D x, S x)=0$ выделяет подпространство в $\Gamma$. Отсюда следует, что $\widehat{\Gamma}(\Gamma)$ - подпространство в $\Gamma$.

Рассмотрим последовательность, порожденную подпространством Г. Положим

$$
\Gamma_{1}=\widehat{\Gamma}(\Gamma), \Gamma_{2}=\widehat{\Gamma}\left(\Gamma_{1}\right), \ldots, \Gamma_{k+1}=\widehat{\Gamma}\left(\Gamma_{k}\right), \ldots
$$

Так как $\Gamma_{k+1} \subset \Gamma_{k}$, то начиная с некоторого $k=k_{0}$ получаем:

$$
\Gamma_{k_{0}+1}=\Gamma_{k_{0}} \text {, откуда } \Gamma_{k}=\Gamma_{k_{0}} \quad \forall k \geqslant k_{0} .
$$

Подпространство $\Gamma_{k_{0}}$ обозначим через $\widetilde{\Gamma}(\Gamma)$. Если $\Gamma=\mathbb{R}^{d(x)}$, то мы будем просто писать $\widetilde{\Gamma}$, опуская аргумент Г. Имеет место

Лемма 3. Для любого Г выполнены условия

1) $f(\widetilde{\Gamma}(\Gamma))=f(\Gamma)$,

2) $\operatorname{Arg} \min f(S, \widetilde{\Gamma}(\Gamma)) \neq \varnothing$. 
ДокАЗАТЕЛьСтво разобьем на ряд этапов.

Сначала докажем, что для любого Г

$$
f(\widehat{\Gamma}(\Gamma))=f(\Gamma) .
$$

Если $\widehat{\Gamma}(\Gamma)=\Gamma$, то это утверждение тривиально. Пусть $\widehat{\Gamma}(\Gamma) \neq \Gamma$. Тогда существует $\widehat{S} \in \Omega(\Gamma)$ такое, что $(D x, \widehat{S} x)<0$ на $\Gamma \backslash \widehat{\Gamma}(\Gamma)$. Отсюда следует формула, справедливая для любого $S$ :

$$
f(S, \widehat{\Gamma}(\Gamma))=\lim _{\lambda \rightarrow \infty} f(S+\lambda \widehat{S}, \Gamma)
$$

из которой следует неравенство:

$$
f(\widehat{\Gamma}(\Gamma)) \geqslant f(\Gamma) .
$$

Обратное неравенство очевидно. Тем самым (3.3) доказано, откуда сразу следует утверждение 1) леммы.

Докажем 2). Если $\widetilde{\Gamma}(\Gamma)=\{0\}$, то утверждение тривиально выполнено, ибо по определению $f(S,\{0\})=-\infty$ для любого $S$ и, следовательно, $\operatorname{Arg} \min f(\{0\})=\mathscr{J}$. Прежде чем перейти к случаю нетривиального $\widetilde{\Gamma}(\Gamma)$, установим эквивалентность:

$$
\widehat{\Gamma}(\Gamma)=\Gamma \Leftrightarrow \Omega(\Gamma)=\{0\} .
$$

Действительно, если $\Omega(\Gamma)=\{0\}$, то по определению $\widehat{\Gamma}(\Gamma)=\Gamma$. Если же $\widehat{\Gamma}(\Gamma)=\Gamma$, то также согласно определению $\Omega(\Gamma) \subset \mathscr{J}_{0}(\Gamma)$ и, следовательно, $\Omega(\Gamma)=\{0\}$. Тем самьм, (3.4) доказано.

Теперь допустим, что $\widetilde{\Gamma}(\Gamma) \neq\{0\}$. Так как $\widehat{\Gamma}(\widetilde{\Gamma}(\Gamma))=\widetilde{\Gamma}(\Gamma)$, то согласно $(3.4)$ $\Omega(\widetilde{\Gamma}(\Gamma))=\{0\}$. Пусть $\left\{S_{n}\right\}$ - минимизирующая последовательность для $f(\widetilde{\Gamma}(\Gamma))$. Если $\left\{S_{n}\right\}$ ограничена, то утверждение 2) леммы верно. Пусть последовательность $\left\{S_{n}\right\}$ неограничена. Без уменьшения общности можно считать, что $S_{n} \in$ $\mathscr{J}_{1}(\widetilde{\Gamma}(\Gamma))$ для любого $n$. Это следует из элементарной формулы: пусть

$$
S=S^{\prime}+S^{\prime \prime}, \quad S^{\prime} \in \mathscr{J}_{0}(\widetilde{\Gamma}(\Gamma)), \quad S^{\prime \prime} \in \mathscr{J}_{1}(\widetilde{\Gamma}(\Gamma)),
$$

тогда

$$
\omega(x, S x)=\omega\left(x, S^{\prime \prime} x\right) \text { на } \Gamma .
$$

Далее, не уменьшая общности, можно считать, что $\left\|S_{n}\right\| \rightarrow \infty$ и $S_{n} /\left\|S_{n}\right\| \rightarrow \widehat{S}$. Очевидно, $\widehat{S} \in \mathscr{J}_{1}(\Gamma)$ и $\|\widehat{S}\|=1$. Докажем, однако, что

$$
\widehat{S} \in \Omega(\widetilde{\Gamma}(\Gamma)) .
$$

Действительно, если сушествует элемент $x \in \widetilde{\Gamma}(\Gamma)$ такой, что $(E \widehat{S} x, \widehat{S} x)>0$, то $f\left(S_{n}, \widetilde{\Gamma}(\Gamma)\right) \rightarrow \infty$ при $n \rightarrow \infty$, что невозможно. Следовательно, $(E \widehat{S} x, \widehat{S} x)=0$ на $\widetilde{\Gamma}(\Gamma)$. Далее, если существует $x \in \widetilde{\Gamma}(\Gamma)$ такой, что $(D x, \widehat{S} x)>0$, то также $f\left(S_{n}, \widetilde{\Gamma}(\Gamma)\right) \rightarrow \infty$. Отсюда $(D x, \widehat{S} x) \leqslant 0$ на $\widetilde{\Gamma}(\Gamma)$. Но тогда $\widehat{S} \in \Omega(\widetilde{\Gamma}(\Gamma))$. Таким образом, (3.5) доказано. Следовательно, $\Omega(\widetilde{\Gamma}(\Gamma)) \neq\{0\}$. Мы пришли к противоречию, откуда следует, что последовательность $\left\{S_{n}\right\}$ ограничена. Лемма доказана.

Возвращаясь к задаче 1 , рассмотрим квадратичную форму

$$
\omega(x, y)=\left(A x+B^{*} y\right)^{2}-2 K x y-Q x x .
$$

Имеем: $\omega(x, S x)=-\omega_{S}(x)$. Форма $\omega(x, y)$ удовлетворяет требованиям леммы 3. 
Лемма 4. Пусть $\Omega\left(\mathbb{R}^{d(x)}\right) \neq\{0\}$. Тогда система $\dot{x}=K x+$ Ви не обладает управляемостью.

ДоКАЗАТЕЛЬСТво. Положим $\Gamma_{0}=\left\{y: B^{*} y=0\right\}$. Из определения $\Omega\left(\mathbb{R}^{d(x)}\right)$ следует, что $S x \in \Gamma_{0} \forall S \in \Omega\left(\mathbb{R}^{d(x)}\right), \forall x \in \mathbb{R}^{d(x)}$. Отсюда следует, что $\Gamma_{0}$ и $\Gamma_{0}^{\prime}$ инвариантные подпространства $S$, причем

$$
S \Gamma_{0}^{\prime}=\{0\}
$$

где $\Gamma_{0}^{\prime}$ - ортогональное дополнение к $\Gamma_{0}$ в $\mathbb{R}^{d(x)}$. Из условия леммы следует:

$$
\Gamma_{0}^{\prime} \neq \mathbb{R}^{d(x)} .
$$

Положим $\Gamma_{n}^{\prime}=K^{n} \Gamma_{0}^{\prime}, n=1,2, \ldots$. Докажем, что $\forall n$

$$
S x \perp \Gamma_{n}^{\prime} \quad \forall S \in \Omega\left(\mathbb{R}^{d(x)}\right), \quad \forall x \in \mathbb{R}^{d(x)} .
$$

Действительно, из (3.6) следует, что (3.8) верно для $n=0$. Пусть (3.8) верно для некоторого $n$. Полагая, что $\Gamma_{n}$ - ортогональное дополнение к $\Gamma_{n}^{\prime}{ }^{\text {в }} \mathbb{R}^{d(x)}$, получаем:

$$
S x \in \Gamma_{n} \quad \forall S \in \Omega\left(\mathbb{R}^{d(x)}\right), \quad \forall x \in \mathbb{R}^{d(x)} .
$$

Элементы $\Gamma_{n}$ обозначим через $\xi$, а элементы $\Gamma_{n}^{\prime}$ - через $\zeta$. По определению конуса $\Omega\left(\mathbb{R}^{d(x)}\right)$ для $S$ вьполнено неравенство

$$
(S x, K x) \geqslant 0 \quad \forall x \in \mathbb{R}^{d(x)} .
$$

Тогда

$$
(S \xi, K \xi)+(S \xi, K \zeta)=0 \quad \forall \xi, \zeta,
$$

откуда, в свою очередь, следует

$$
(S \xi, K \zeta)=0 \quad \forall \xi, \zeta .
$$

Учитывая, что $S \Gamma_{n}^{\prime}=0$, получаем отсюда

$$
\left(S x, K \Gamma_{n}^{\prime}\right)=0 \quad \forall x \in \mathbb{R}^{d(x)} .
$$

Но $K \Gamma_{n}^{\prime}=\Gamma_{n+1}^{\prime}$. Таким образом, мы установили, что $S x \perp \Gamma_{n+1}^{\prime} \forall x$.

Отсюда согласно индукции следует (3.8).

Положим $\bar{\Gamma}_{n}^{\prime}=\sum_{k=0}^{n} \Gamma_{k}^{\prime}$. Через $\bar{\Gamma}_{n}$ обозначим ортогональное дополнение к $\bar{\Gamma}_{n}^{\prime}$ в $\mathbb{R}^{d(x)}$. Очевидно, что $\bar{\Gamma}_{n}^{\prime}$ - неубьвающая последовательность подпространств, соответственно $\bar{\Gamma}_{n}$ - невозрастающая последовательность подпространств.

Положим $\bar{\Gamma}_{\infty}=\bigcap_{n} \bar{\Gamma}_{n}$. Докажем, что

$$
\bar{\Gamma}_{\infty} \neq\{0\} .
$$

Действительно, допустив противное, получим согласно (3.8) $S=0$, что противоречит условию леммы. Тем самым (3.9) доказано. Полагая, что $\bar{\Gamma}_{\infty}^{\prime}-$ ортогональное дополнение к $\bar{\Gamma}_{\infty}$ в $\mathbb{R}^{d(x)}$, получаем из (3.8)

$$
K \bar{\Gamma}_{\infty}^{\prime} \subset \bar{\Gamma}_{\infty}^{\prime}
$$


Кроме того, из $\bar{\Gamma}_{\infty} \subset \Gamma_{0}$ следует

$$
B^{*} \bar{\Gamma}_{\infty}=\{0\}
$$

Обозначая элементы $\bar{\Gamma}_{\infty}$ через $\xi$, а элементы $\bar{\Gamma}_{\infty}^{\prime}$ через $\zeta$, получаем

$$
\dot{\xi}=P_{\infty} K \xi+P_{\infty} K \zeta+P_{\infty} B u
$$

где $P_{\infty}$ - ортогональньй проектор на $\bar{\Gamma}_{\infty}$. Тогда согласно $(3.10)$ и (3.11) получим: $\dot{\xi}=P_{\infty} K \xi$. Но это и означает неуправляемость системы $\dot{x}=K x+B u$. Лемма доказана.

Итак, если система $V: \dot{x}=K x+B u$ управляема, то по лемме $4 \Omega\left(\mathbb{R}^{d(x)}\right)=\{0\}$ и, следовательно, $\widetilde{\Gamma}=\mathbb{R}^{d(x)}$ (по лемме 3 ). С другой стороны, любая управляемая система $V$ разбивается на две подсистемы: первая представляет собой замкнутое дифференциальное уравнение, не содержащее управления, а вторая обладает управляемостью. Конечно, может случиться так, что одна из подсистем отсутствует. Компоненты, входящие в подсистему первого типа, равны тождественно нулю на $L(V)$ в силу нулевых начальных условий. Поэтому фактически мы будем иметь дело с управляемой системой. Таким образом, требование управляемости не снижает общности рассмотрения.

\section{§4. Доказательство теоремы 1. Двойственность}

4.1. Прежде всего установим, что из неравенства $\widehat{\lambda} \geqslant 0$ следует неотрицательность $J$ на $L(V)$. Применяя лемму 4 к форме

$$
\omega(x, y)=\left(A x+B^{*} y\right)^{2}-2 K x y-Q x x
$$

и учитывая, что система $V$ обладает управляемостью, получаем, что существует $\widehat{S}$ такое, что

$$
\lambda(\widehat{S})=\widehat{\lambda}
$$

Тогда $\Phi_{\widehat{S}}(x, u) \geqslant 0 \forall x, u$, откуда следует, что $J \geqslant 0$ на $L(V)$.

Прежде чем перейти к рассмотрению случая $\widehat{\lambda}<0$, докажем следуюшее предложение. Назовем әлементарным ииклом траекторию системы $V$ вида:

$$
\begin{aligned}
& x(t)=x_{0} \cos \sigma t+x_{1} \sin \sigma t, \\
& u(t)=u_{0} \cos \sigma t+u_{1} \sin \sigma t,
\end{aligned}
$$

где $\left(x_{0}, x_{1}\right) \neq 0, x_{0} \perp x_{1}$. Через $T_{\sigma}$ будем обозначать любой период элементарного цикла.

ПРЕДЛОЖЕНИЕ 1. 1) Для любого әлементарного иикла справедливо неравенство

$$
\frac{\int_{0}^{T_{\sigma}} \Phi(x(t), u(t)) d t}{\int_{0}^{T_{\sigma}} x^{2}(t) d t} \geqslant \widehat{\lambda}
$$

2) Существует әлементарный цикл, для которого в (*) въполнено равенство. 
ДокАЗАТЕльство. Отметим, что отношение в левой части рассматриваемого выражения не зависит от $T_{\sigma}$. Прежде всего, ясно, что

$$
\int_{0}^{T_{\sigma}} \Phi(x(t), u(t)) d t=\int_{0}^{T_{\sigma}} \Phi_{S}(x(t), u(t)) d t
$$

для любого элементарного цикла, любого его периода $T_{\sigma}$ и любого $S$. Следовательно,

$$
\frac{\int_{0}^{T_{\sigma}} \Phi(x(t), u(t)) d t}{\int_{0}^{T_{\sigma}} x^{2}(t) d t} \geqslant \frac{\int_{0}^{T_{\sigma}} \omega_{\widehat{S}}(x) d t}{\int_{0}^{T_{\sigma}} x^{2}(t) d t}
$$

но $\omega_{\widehat{S}}(x) \geqslant \widehat{\lambda} x^{2}$. Таким образом, утверждение 1) предложения 1 доказано.

Воспользуемся тем, что

$$
\lambda(\widehat{S})=\max _{S} \lambda(S)
$$

Необходимое условие, которому должно удовлетворять $\widehat{S}$, имеет следующий вид:

$$
\min _{x \in \Sigma_{0}}\left(R_{\widehat{S}} x, \bar{S} x\right) \leqslant 0 \quad \forall \bar{S}
$$

где $R_{S}=K+B U_{S}$

$$
\begin{aligned}
& \Gamma_{0}=\left\{x: \omega_{\widehat{S}}(x)=\widehat{\lambda} x^{2}\right\} \\
& \Sigma_{0}=\left\{x \in \Gamma_{0}:|x|=1\right\} .
\end{aligned}
$$

Иными словами, для $-R_{\widehat{S}}$ выполнено условие $\left(* ; \Gamma_{0}\right)$, см. $\S 3$. Согласно лемме 2 сушествует подпространство $\Gamma_{1}$ :

$$
\Gamma_{1} \subset \Gamma_{0}, \quad d\left(\Gamma_{1}\right)>0, \quad R_{\widehat{S}} \Gamma_{1} \subset \Gamma_{1}
$$

и $R_{\widehat{S}}$ на $\Gamma_{1}$ имеет собственное число с нулевой вещественной частью. Следовательно, в $\Gamma_{1}$ существует $\Gamma \subset \Gamma_{1}$, инвариантное относительно $R_{\widehat{S}}$, такое, что $d(\Gamma)=1$ или 2. Таким образом, $\Gamma$ - собственное подпространство $R_{\widehat{S}}$, соответствуюшее этому собственному числу. Если $d(\Gamma)=1$, то собственное число $R_{\widehat{S}}$ на $\Gamma$ равно нулю. Если $d(\Gamma)=2$, то $R_{\widehat{S}}$ на $Г$ имеет пару сопряженных собственных чисел $\pm i \sigma, \sigma \neq 0$. Отсюда следует, что $R_{\widehat{S}} \Gamma=0$, если $d(\Gamma)=1$. В случае $d(\Gamma)=2$ оператор $R_{\widehat{S}}$ имеет следующее представление:

$$
R_{\widehat{S}} x_{0}=\sigma x_{1}, \quad R_{\widehat{S}} x_{1}=-\sigma x_{0}
$$

где

$$
\operatorname{Lin}\left(x_{0}, x_{1}\right)=\Gamma, \quad\left(x_{0}, x_{1}\right)=0 .
$$

Для определенности рассмотрим последний случай. Пусть $x^{0}(t)$ есть решение уравнения:

$$
\frac{d x}{d t}=R_{\widehat{S}} x, \quad x^{0}(0)=x_{0}
$$


Полагая $u_{0}^{0}(t)=U_{\widehat{S}} x^{0}(t)$, получаем, что пара $x(t), u(t)$ является элементарным циклом, представимым в виде:

$$
\begin{aligned}
& x(t)=x_{0} \cos \sigma t+x_{1} \sin \sigma t, \\
& u(t)=u_{0} \cos \sigma t+u_{1} \sin \sigma t,
\end{aligned}
$$

где $x_{0}, x_{1}$ введены в (4.1), $u_{0}=U_{\widehat{S}} x_{0}, u_{1}=U_{\widehat{S}} x_{1}$. Имеем:

$$
\Phi_{\widehat{S}}\left(x^{0}(t), u^{0}(t)\right)=\omega_{\widehat{S}}\left(x^{0}(t)\right)=\widehat{\lambda}\left(x^{0}(t)\right)^{2} .
$$

Отсюда следует утверждение 2) предложения 1 . Тем самым оно доказано.

Продолжим доказательство теоремы 1 и рассмотрим случай $\widehat{\lambda}<0$.

Пусть $x(t), u(t)$ - элементарный цикл такой, что

$$
\frac{\int_{0}^{T_{\sigma}} \Phi(x(t), u(t)) d t}{\int_{0}^{T_{\sigma}} x^{2}(t) d t}=\widehat{\lambda} .
$$

Поскольку система $V$ управляема, то можно за конечное время перейти из нуля на фазовую компоненту цикла, пробыть на нем любое время, равное некоторому $T_{\sigma}$, и вновь за конечное время вернуться в нуль.

Для достаточно больших $T_{\sigma}$ мы получим траекторию из $L(V)$, на которой $J<0$. Тем самым теорема 1 доказана.

4.2. Предложение 1 устанавливает связь между частотньм критерием и критерием, основанньм на неравенстве Гамильтона-Якоби. Эта связь представляет собой некоторую теорему двойственности.

Пусть $x(t), u(t)$ - периодическая траектория системы $V$, и пусть $\{T\}$ - множество ее периодов. Положим

$$
Z(\Phi)=\frac{\int_{0}^{T} \Phi(x(t), u(t)) d t}{\int_{0}^{T} x^{2}(t) d t}, \quad T \in\{T\} .
$$

Очевидно, что $Z(\Phi)$ не зависит от выбора $T$ и представляет собой линейный функционал на множестве функций $\Phi(x, u)$ рассматриваемого вида (см. $\S 1)$, а это множество - обозначим его через $\widetilde{\Phi}$ - вьпукло. Таким образом, каждый нетривиальный цикл порождает линейный функционал на выпуклом множестве $\widetilde{\Phi}$. Рассмотрим на $\widetilde{\Phi}$ функционал:

$$
Y(\Phi)=\min \left\{\frac{\Phi(x, u)}{x^{2}}: u \in \mathbb{R}^{d(u)}, x \in \mathbb{R}^{d(x)} \backslash\{0\}\right\} .
$$

Заметим, что из $\Phi \in \widetilde{\Phi}$ следует, что $\Phi_{S} \in \widetilde{\Phi} \forall S$. Кроме того, для $S=0$ имеем $\Phi_{S}=\Phi$. Обозначим периодическую нетривиальную траекторию системы $V$ через $\zeta$. 
ПРЕДЛОЖЕНИЕ 2. Имеет место следующая формула двойственности:

$$
\min _{\zeta} Z(\Phi)=\max _{S} Y\left(\Phi_{S}\right) \quad \text { для любого } \quad \Phi \in \widetilde{\Phi} .
$$

ДокАЗАТЕЛЬСТво. Имеем согласно $\S 2$ :

$$
\Phi_{S}(x, u)=\left(u-U_{S} x\right)^{2}+\omega_{S}(x)
$$

Отсюда следует, что для любого $\zeta$ выполнено $Z\left(\Phi_{S}\right) \geqslant \lambda(S)$. Тогда, поскольку $Z\left(\Phi_{S}\right)=Z(\Phi)$, имеем:

$$
Z(\Phi) \geqslant \lambda(S)
$$

Следовательно, для любого $\zeta$

$$
Z(\Phi) \geqslant \widehat{\lambda}
$$

С другой стороны, $Y\left(\Phi_{S}\right)=\lambda(S)$, откуда $\widehat{\lambda}=\max _{S} Y\left(\Phi_{S}\right)$. Тем самым доказано неравенство

$$
\min _{\zeta} Z(\Phi) \geqslant \max _{S} Y\left(\Phi_{S}\right)
$$

Из предложения 1 следует, что существует элементарный цикл $\zeta^{0}$ такой, что $Z(\Phi)=\widehat{\lambda}$, а тогда справедливо равенство (4.2). Предложение 2 доказано.

Рассмотрим теперь частотный критерий [1]. Положим

$$
f=u_{0}^{2}+u_{1}^{2}+2\left(A x_{0} u_{0}+A x_{1} u_{1}\right)+Q x_{0} x_{0}+Q x_{1} x_{1}
$$

Частотный критерий состоит в следующем. Для того чтобы выполнялось неравенство $J \geqslant 0$ на $L(V)$, необходимо и достаточно, чтобы выполнялось неравенство $f \geqslant 0$ для любых $\sigma, x_{0}, x_{1}, u_{0}, u_{1}$ таких, что

$$
\begin{aligned}
\sigma x_{1} & =K x_{0}+B u_{0}, \\
-\sigma x_{0} & =K x_{1}+B u_{1} .
\end{aligned}
$$

Предложение 1 можно записать в форме:

$$
\min \frac{f}{x_{0}^{2}+x_{1}^{2}}=\widehat{\lambda}
$$

где min берется по всем $\sigma, x_{0}, x_{1}, u_{0}, u_{1}$, удовлетворяющим (4.4). Действительно, для любого элементарного цикла верно равенство:

$$
\frac{f}{x_{0}^{2}+x_{1}^{2}}=\frac{\int_{0}^{T_{\sigma}} \Phi(x(t), u(t)) d t}{\int_{0}^{T_{\sigma}} x^{2}(t) d t} \forall T_{\sigma} .
$$

Отсюда и из предложения 1 легко следует (4.5). Таким образом, частотный критерий следует из теоремы 1 и не является единственньм аппаратом исследования интегральных квадратичных форм с постоянными коэффициентами на полуоси.

В следуюших двух параграф̆ах мы приведем некоторые примеры применения теоремы 1 и предложения 1. 


\section{§5. Квадратичное регулирование}

5.1. Пусть задана управляемая система $V: \dot{x}=K x+B u$, обладающая управляемостью в смысле $\S 3$. Положим $\mathscr{U}=\pi_{u(\cdot)} L(V), \mathscr{X}=\pi_{x(\cdot)} L(V)$. На линейных многообразиях $\mathscr{U}, \mathscr{X}$ введем нормы

$$
\|u(\cdot)\|=\sqrt{\int_{0}^{\infty} u^{2}(t) d t}, \quad\|x(\cdot)\|=\sqrt{\int_{0}^{\infty} x^{2}(t) d t}
$$

и рассмотрим оператор

$$
F: \mathscr{U} \rightarrow \mathscr{X}, \quad u(\cdot) \rightarrow x(\cdot), \quad \text { где }(u(t), x(t)) \in L(V)
$$

Поставим вопрос: когда оператор $F$ ограничен и какова его норма?

Положим $\Phi=u^{2}$. Для любого $S$ согласно $\S 2$ имеем:

$$
\int_{0}^{\infty} \Phi d t=\int_{0}^{\infty}\left[\left(u-U_{S} x\right)^{2}+\omega_{S}(x)\right] d t
$$

Пусть $\widehat{\lambda}=\max _{S} \lambda(S)$. Так как при $S=0$ имеем $\lambda(S)=0$, то $\hat{\lambda} \geqslant 0$. Справедлива следующая

ТЕорема 2. Oператор F ограничен тогда и только тогда, когда $\widehat{\lambda}>0$. Его норма равна $1 / \sqrt{\widehat{\lambda}}$.

ДоказАтЕЛЬство. Пусть $\widehat{\lambda}>0$ и $\widehat{S}$ таково, что $\lambda(\widehat{S})=\widehat{\lambda}$. Имеем:

$$
\Phi_{\widehat{S}}(x, u)-\widehat{\lambda} x^{2}=\left(u-U_{\widehat{S}} x\right)^{2}+\omega_{\widehat{S}}(x)-\widehat{\lambda} x^{2} \geqslant 0
$$

Следовательно,

$$
J_{\widehat{S}}(x(\cdot), u(\cdot)) \geqslant \widehat{\lambda} \int_{0}^{\infty} x^{2}(t) d t \text { на } L(V),
$$

но на $L(V)$ выполнено $J_{\widehat{S}}=J$. Следовательно,

$$
\int_{0}^{\infty} u^{2} d t \geqslant \widehat{\lambda} \int_{0}^{\infty} x^{2} d t
$$

откуда $\|F\| \leqslant 1 / \sqrt{\widehat{\lambda}}$. С другой стороны, по предложению 1 сушествует элементарный цикл $x^{0}(t), u^{0}(t)$ такой, что

$$
\int_{0}^{T_{\sigma}}\left(u^{0}(t)\right)^{2} d t=\widehat{\lambda} \int_{0}^{T_{\sigma}}\left(x^{0}(t)\right)^{2} d t
$$

откуда $\|F\| \geqslant 1 / \sqrt{\widehat{\lambda}}$. Таким образом,

$$
\widehat{\lambda}>0 \Rightarrow\|F\|=\frac{1}{\sqrt{\widehat{\lambda}}}
$$


Пусть $\widehat{\lambda}=0$. Согласно предложению 1 сушествует элементарный цикл $x^{0}(t), u^{0}(t)$ такой, что $u^{0}(t)=0$. Отсюда следует неограниченность $F$. Теорема доказана.

Имеем далее $\omega_{S}(x)=-\left(B^{*} S x\right)^{2}+2(K x, S x)$. Следовательно, $\widehat{\lambda}>0$ тогда и только тогда, когда существует $S$ такое, что

$$
(K x, S x)>0 \quad \forall x \neq 0 .
$$

По лемме 1 это эквивалентно условию: $K$ не имеет собственных чисел с нулевой вещественной частью. Таким образом, оператор $F$ ограничен тогда и только тогда, когда $K$ не имеет собственных чисел с нулевой вешественной частью.

Замькание $\mathscr{U}$ по норме $L_{2}$ является подпространством конечной коразмерности, которая равна числу собственных чисел $K$ с неотрицательной вещественной частью. Если таковых нет, то замыкание $\mathscr{U}$ есть все пространство. Следовательно, для того чтобы решение $x(t)$ уравнения $\dot{x}=K x+B u, x(0)=0$, принадлежало $L_{2}$, коль скоро $u(t) \in L_{2}$, необходимо и достаточно, чтобы все собственные числа $K$ имели отрицательную вешественную часть.

5.2. Пусть заданы $\Phi$ и $V$. Рассмотрим задачу:

$$
\begin{gathered}
J=\int_{0}^{\infty} \Phi d t \rightarrow \inf , \quad \dot{x}=K x+B u, \quad x(0)=x_{*}, \\
\int_{0}^{\infty}\left(x^{2}+u^{2}\right) d t<+\infty .
\end{gathered}
$$

Предполагаем по-прежнему, что система $V$ обладает управляемостью.

В обзоре [1] приведена следуюшая классификация. Возможны три случая:

1) $\inf J=-\infty \forall x_{*}$;

$2) \inf J=\min J \forall x_{*}$ и $\operatorname{Arg} \min J$ состоит из одной точки (регулярный случай);

$3) \inf J>-\infty \forall x_{*}$, но регулярности нет.

Мы докажем эту классификацию, не опираясь на частотный критерий.

Tеорема 3. Случай $\inf J=-\infty \quad \forall x_{*}$ әквивалентен неравенству $\widehat{\lambda}<0$.

Случай $\operatorname{Arg} \min J \neq \varnothing \quad \forall x_{*}$ әквивалентен неравенству $\widehat{\lambda}>0$. $\widehat{\lambda}=0$.

Случай $\inf J>-\infty \quad \forall x_{*} u \exists x_{*}: \operatorname{Arg} \min J=\varnothing$ әквивалентен равенству

ДокАЗАТЕльство. Пусть $\widehat{\lambda}<0$. По теореме 1 в этом случае существует траектория $\left(x^{0}(t), u^{0}(t)\right) \in L(V)$ такая, что

$$
J\left(x^{0}(\cdot), u^{0}(\cdot)\right)<0 .
$$

Тогда для любой траектории $\left(x^{1}(t), u^{1}(t)\right)$, допустимой в задаче $(5.2)$, траектория $\left(x^{1}(t)+\tau x^{0}(t), u^{1}(t)+\tau u^{0}(t)\right)$ также будет допустима для любого $\tau$ и $J\left(x^{1}+\tau x^{0}\right.$, $\left.u^{1}+\tau u^{0}\right) \rightarrow-\infty$ при $\tau \rightarrow \infty$. Следовательно, $\inf J=-\infty \forall x_{*}$.

Пусть $\widehat{\lambda}>0$. Возьмем $\widehat{S}$ таким, чтобы $\lambda(\widehat{S})=\widehat{\lambda}$, и положим

$$
J_{\widehat{S}}=\int_{0}^{\infty}\left(\Phi+\frac{d}{d t}(\widehat{S} x x)\right) d t=J-\left(\widehat{S} x_{*} x_{*}\right) .
$$


Имеем:

$$
J_{\widehat{S}}=\int_{0}^{\infty}\left[\left(u-U_{\widehat{S}} x\right)^{2}+\omega_{\widehat{S}}(x)\right] d t \geqslant \int_{0}^{\infty}\left[\left(u-U_{\widehat{S}} x\right)^{2}+\widehat{\lambda} x^{2}\right] d t
$$

для любой траектории, допустимой в задаче (5.2). Отсюда следует, что существует $\delta>0$ такое, что

$$
J_{\widehat{S}} \geqslant \delta \int_{0}^{\infty}\left[x^{2}(t)+u^{2}(t)\right] d t .
$$

Тогда $\operatorname{Arg} \min J_{\widehat{S}}$ непусто $\forall x_{*}$ и содержит ровно один элемент. Но $\operatorname{Arg} \min J_{\widehat{S}}=$ $\operatorname{Arg} \min J$.

Пусть $\widehat{\lambda}=0$. Тогда $J_{\widehat{s}} \geqslant 0$ для любой допустимой траектории и, следовательно, inf $J>-\infty \forall x_{*}$. Согласно предложению 1 сушествует элементарный цикл такой, что $\int_{0}^{T} \Phi d t=0$ для любого периода $T$. Пусть этот цикл есть $x^{0}(t), u^{0}(t)$. Возьмем $x_{*}=x^{0}(0)$. Допустим, что для $x_{*} \operatorname{Arg} \min J \neq \varnothing$. Тогда $\operatorname{Arg} \min J$ содержит более чем одну траекторию. Однако нетрудно доказать, что если $\operatorname{Arg} \min J \neq \varnothing$, то он состоит из одной точки. Действительно, пусть

$$
\left(x^{1}(t), u^{1}(t)\right) \in \operatorname{Arg} \min J \text { и }\left(x^{2}(t), u^{2}(t)\right) \in \operatorname{Arg} \min J .
$$

Так как $\widehat{\lambda}=0$, то $J_{\widehat{S}}$ - выпуклый функционал на допустимых траекториях задачи (5.2). Положим $x^{1}-x^{2}=\delta x, u^{1}-u^{2}=\delta u$. Тогда необходимо должно выполняться равенство

$$
J_{\widehat{S}}(\delta x(\cdot), \delta u(\cdot))=0 .
$$

Отсюда $\delta u=U_{\widehat{S}} \delta x$ и тогда $\delta x=0, \delta u=0$ в силу системы $V$. Следовательно, $\operatorname{Arg} \min J$ может состоять не более чем из одной допустимой траектории. Но тогда для данного $x_{*} \operatorname{Arg} \min J=\varnothing$. Теорема доказана.

\section{§6. Необходимые и достаточные условия неотрицательности формы $J$ на $L(V)$, порожденные теоремой 1}

6.1. Условия, необходимые и достаточные для неотрицательности формы $J$ на $L(V)$, мы будем впредь коротко называть условиями неотрицательности. Непосредственно из доказательства теоремы вытекают следующие условия неотрицательности.

Назовем пару $\Gamma, S$ согласованной, если выполнены следующие условия:

$$
\begin{aligned}
& d(\Gamma) \in\{1,2\} ; \\
& R_{S} \Gamma \subset \Gamma ; \\
& \text { собственные числа } R_{S} \text { на } \Gamma \text { равны } 0, \text { если } d(\Gamma)=1, \\
& \text { и равны } \pm i \sigma, \sigma \neq 0, \text { если } d(\Gamma)=2 ; \\
& \omega_{S}(x)=\kappa x^{2} \text { на } \Gamma ; \\
& \omega_{S}^{\prime}\left(x, x^{\prime}\right)=0 \forall x \in \Gamma, x^{\prime} \perp \Gamma .
\end{aligned}
$$

Тогда из доказательства теоремы 1 вытекает непосредственно, что следующее условие является условием неотрицательности. 
УСЛОВИЕ 1. Для любой согласованной пары имеет место неравенство $\kappa \geqslant 0$.

Мы скажем, что пара $\Gamma, S$ слабо согласована, если выполнены условия $\left(6.1_{1}\right)-$ $\left(6.1_{4}\right)$. Следующее условие также является условием неотрицательности.

УСЛОВИЕ 2. Для любой слабо согласованной пары имеет место неравенство $\kappa \geqslant 0$.

Это условие также непосредственно следует из доказательства теоремы 1 . Ниже мы расшифруем условия 1 и 2.

6.2. Содержанием этого пункта является доказательство формулы

$$
\frac{1}{2} \omega_{S}^{\prime}(x, y)=\left(U_{S} x, A y\right)+\left(S R_{S} x, y\right)+(S x, K y)+(Q x, y)
$$

справедливой для любых $x, y$. Здесь $\omega_{S}^{\prime}(x, y)$ - производная формы $\omega_{S}$ в точке $x$ по направлению $y$ :

$$
\omega_{S}^{\prime}(x, y)=\left.\frac{d}{d \varepsilon} \omega_{S}(x+\varepsilon y)\right|_{\varepsilon=0} .
$$

Имеем согласно $\S 2$ :

$$
\omega_{S}(x)=-\left(U_{S} x\right)^{2}+2(K x, S x)+(Q x, x) .
$$

Следовательно,

$$
\frac{1}{2} \omega_{S}^{\prime}(x, y)=-\left(U_{S} x, U_{S} y\right)+(K x, S y)+(S x, K y)+(Q x, y)
$$

По определению $U_{S}=-\left(A+B^{*} S\right)$. Следовательно,

$$
-\left(U_{S} x, U_{S} y\right)=\left(U_{S} x, A y\right)+\left(U_{S} x, B^{*} S y\right) .
$$

С другой стороны,

$$
(K x, S y)=\left(S R_{S} x, y\right)-\left(S B U_{S} x, y\right),
$$

что следует из определения $R_{S}: R_{S}=K+B U_{S}$. Учитывая два последних равенства, получаем:

$$
\frac{1}{2} \omega_{S}^{\prime}(x, y)=\left(U_{S} x, A y\right)+\left(S R_{S} x, y\right)+(S x, K y)+(Q x, y)
$$

что и требовалось.

6.3. Пусть $d(\Gamma)=1$. Выясним, когда сушествует $S$, слабо согласованное с $\Gamma$.

Теорема 4. Слабо согласованная с $Г$ матрица $S$ существует тогда и только тогда, когда существуют $\widehat{x}, \widehat{p}, \widehat{u}$ такие, что:

1) $\widehat{x} \in \Gamma, \widehat{x} \neq 0$;

2) $0=K \widehat{x}+B \widehat{u}$;

3) $\widehat{u}=-\left(A \widehat{x}+B^{*} \widehat{p}\right)$. 
ДоказАТЕльство. Пусть $S$ таково, что пара $\Gamma, S$ слабо согласована. Выберем $\widehat{x} \in \Gamma, \widehat{x} \neq 0$, и положим $\widehat{p}=S \widehat{x}$. По определению слабой согласованности $R_{S} \widehat{x}=0$. Следовательно, полагая $\widehat{u}=-\left(A \widehat{x}+B^{*} \widehat{p}\right)$, получаем $0=K \widehat{x}+B \widehat{u}$. Необходимость условий теоремы доказана.

Пусть теперь существуют $\widehat{x}, \widehat{p}, \widehat{u}$, удовлетворяющие условиям 1$)-3)$ теоремы. Выберем $S$ так, чтобы $S \widehat{x}=\widehat{p}$. Тогда согласно условию 3 )

$$
\begin{gathered}
\widehat{u}=U_{S} \widehat{x}, \\
K \widehat{x}+B \widehat{u}=R_{S} \widehat{x} .
\end{gathered}
$$

Согласно условию 2) отсюда следует, что $R_{S} \widehat{x}=0$. Достаточность условий 1)-3) теоремы, а вместе с ней и вся теорема доказаны.

Выясним, когда сушествует $S$, согласованное с $\Gamma$.

Теорема 5. Матрица S, согласованная с Г, существует тогда и только тогда, когда существуют $\widehat{x}, \widehat{p}, \widehat{u}$ такие, что:

1) $\widehat{x} \in \Gamma, \widehat{x} \neq 0$;

2) $0=K \widehat{x}+B \widehat{u}$;

3) $\widehat{u}=-\left(A \widehat{x}+B^{*} \widehat{p}\right)$;

4) $\widehat{u} A+\widehat{p} K+Q \widehat{x}=\kappa \widehat{x}$.

ДоказАТЕльство. Пусть $S$ согласовано с $\Gamma$. Выберем произвольно $\widehat{x} \in \Gamma$, $\widehat{x} \neq 0$. Положим $\widehat{p}=S \widehat{x}, \widehat{u}=U_{S} \widehat{x}$. Очевидно, что $\widehat{x}, \widehat{p}, \widehat{u}$ удовлетворяют условиям $1)-3$ ) теоремы, ибо $R_{S} \widehat{x}=0$ по условию $\left(6.1_{3}\right)$. Далее, согласно $\left(6.1_{5}\right)$ имеем:

$$
\omega_{S}^{\prime}\left(\widehat{x}, x^{\prime}\right)=0 \quad \forall x^{\prime} \perp \widehat{x}
$$

Согласно формуле (6.2) отсюда следует:

$$
\left(\widehat{u}, A x^{\prime}\right)+\left(\widehat{p}, K x^{\prime}\right)+\left(Q \widehat{x}, x^{\prime}\right)=0 \quad \forall x^{\prime} \perp \widehat{x}
$$

откуда вытекает условие 4) теоремы. Необходимость доказана.

Перейдем к доказательству достаточности. Пусть $\widehat{x}, \widehat{p}, \widehat{u}$ удовлетворяют условиям 1)-4) теоремы. Выберем $S$ так, чтобы $S \widehat{x}=\widehat{p}$, и докажем, что $S$ согласовано с Г. Рассуждая так же, как при доказательстве предыдущей теоремы, установим, что $S$ слабо согласовано с Г. Установим (6.15). Согласно формуле (6.2) имеем для $x^{\prime} \perp \widehat{x}$

$$
\frac{1}{2} \omega_{S}^{\prime}\left(\widehat{x}, x^{\prime}\right)=\left(\widehat{u}, A x^{\prime}\right)+\left(S R_{S} \widehat{x}, x^{\prime}\right)+\left(\widehat{p}, K x^{\prime}\right)+\left(Q \widehat{x}, x^{\prime}\right)=\kappa\left(\widehat{x}, x^{\prime}\right)=0
$$

Второе равенство следует из условия 4) теоремы. Тем самым установлено равенство $\left(6.1_{5}\right)$. Достаточность, а вместе с ней и вся теорема доказаны.

6.4. В этом пункте мы выясним тот же вопрос, что и в п. 6.3 , но для двумерного Г. Итак, пусть задано $Г, d(\Gamma)=2$. 
ТеОрема 6. Матрица S, слабо согласованная с Г, существует тогда и только тогда, когда существуют $\sigma, \kappa ; x_{0}, x_{1} ; p_{0}, p_{1} ; u_{0}, u_{1}$ такие, что:

1) $\sigma \neq 0$;

2) $\operatorname{Lin}\left(x_{0}, x_{1}\right)=\Gamma,\left(x_{0}, x_{1}\right)=0$;

3) $p_{1} x_{0}=p_{0} x_{1}$

4) $\sigma x_{1}=K x_{0}+B u_{0},-\sigma x_{0}=K x_{1}+B u_{1}$;

5) $u_{0}=-\left(A x_{0}+B^{*} p_{0}\right), u_{1}=-\left(A x_{1}+B^{*} p_{1}\right)$;

6) $-u_{0}^{2}+2\left(p_{0}, K x_{0}\right)+\left(Q x_{0}, x_{0}\right)=\kappa x_{0}^{2}$, $-u_{1}^{2}+2\left(p_{1}, K x_{1}\right)+\left(Q x_{1}, x_{1}\right)=\kappa x_{1}^{2}$, $-u_{0} u_{1}+\left(p_{1}, K x_{0}\right)+\left(p_{0}, K x_{1}\right)+\left(Q x_{0}, x_{1}\right)=0$.

ДокАЗАТЕльство. Пусть $S$ слабо согласовано с $\Gamma$. В силу $\left(6.1_{2}\right) R_{S}$ имеет $\Gamma$ своим инвариантным подпространством, а согласно $\left(6.1_{3}\right)$ его собственные числа на $Г$ имеют вид $\pm i \sigma, \sigma \neq 0$. Тем самым постоянная $\sigma$ определена. Далее, существует ортогональньй базис $x_{0}, x_{1}$ в $\Gamma$, в котором $R_{S}$ имеет вид

$$
\sigma x_{1}=R_{S} x_{0}, \quad-\sigma x_{0}=R_{S} x_{1}
$$

Тем самым определены векторы $x_{0}, x_{1}$. Заметим, что $\sigma, x_{0}, x_{1}$ удовлетворяют условиям 1), 2) теоремы. Положим, далее, $p_{0}=S x_{0}, p_{1}=S x_{1}, u_{0}=U_{S} x_{0}$, $u_{1}=U_{S} x_{1}$.

Исходя из определений $U_{S}$ и $R_{S}$ нетрудно установить, что $\sigma, x_{0}, x_{1} ; p_{0}, p_{1} ; u_{0}$, $u_{1}$ удовлетворяют условиям 3$)-5$ ) теоремы. Остается показать, что выполняются также и условия 6).

По условию $\left(6.1_{4}\right) \omega_{S}(x)=\kappa x^{2}$ на $\Gamma$. Тем самым постоянная $\kappa$ определена. Очевидно, равенство $\left(6.1_{4}\right)$ эквивалентно равенствам:

$$
\begin{gathered}
\omega_{S}\left(x_{0}\right)=\kappa x_{0}^{2}, \quad \omega_{S}\left(x_{1}\right)=\kappa x_{1}^{2}, \\
\omega_{S}^{\prime}\left(x_{0}, x_{1}\right)=0 \quad\left(\text { ибо } x_{0} \perp x_{1}\right) .
\end{gathered}
$$

Условия 6) теоремы элементарно вытекают из этих равенств. Необходимость доказана.

Перейдем к достаточности. Пусть $\sigma, \kappa ; x_{0}, x_{1} ; p_{0}, p_{1} ; u_{0}, u_{1}$ удовлетворяют условиям 1)-6) теоремы. Определим матрицу $S$ так, чтобы $S x_{0}=p_{0}, S x_{1}=p_{1}$. Эти требования корректны в силу условия 3$)$. Тогда $u_{0}=U_{S} x_{0}, u_{1}=U_{S} x_{1}$. Из условий 4), 5) следует:

$$
\sigma x_{1}=R_{S} x_{0}, \quad-\sigma x_{0}=R_{S} x_{1}
$$

Отсюда следует, что Г инвариантно относительно $R_{S}$ и что собственные числа $R_{S}$ на Г равны $\pm i \sigma$. Из условий 6 ) следует, что

$$
\omega_{S}(x)=\kappa x^{2} \text { на } \Gamma \text {. }
$$

Достаточность доказана. Тем самым доказательство теоремы завершено.

Выясним, когда сушествует $S$, согласованное с $\Gamma$. 
ТЕОРема 7. Матрица S, согласованная с Г, существует тогда и только тогда, когда существуют $\sigma, \kappa ; x_{0}, x_{1} ; p_{0}, p_{1} ; u_{0}, u_{1}$ такие, что:

1) $\sigma \neq 0$;

2) $\operatorname{Lin}\left(x_{0}, x_{1}\right)=\Gamma,\left(x_{0}, x_{1}\right)=0$

3) $p_{1} x_{0}=p_{0} x_{1}$

4) $\sigma x_{1}=K x_{0}+B u_{0},-\sigma x_{0}=K x_{1}+B u_{1}$

5) $u_{0}=-\left(A x_{0}+B^{*} p_{0}\right), u_{1}=-\left(A x_{1}+B^{*} p_{1}\right)$;

6) $u_{0} A+\sigma p_{1}+p_{0} K+Q x_{0}=\kappa x_{0}$, $u_{1} A-\sigma p_{0}+p_{1} K+Q x_{1}=\kappa x_{1}$.

ДоказАТельство. Докажем необходимость. Пусть $S$ согласовано с Г. Тогда $S$ слабо согласовано с $\Gamma$ и, также как и при доказательстве теоремы 6 , мы определим $\sigma, \kappa ; x_{0}, x_{1} ; p_{0}, p_{1} ; u_{0}, u_{1}$, удовлетворяющие всем условиям теоремы 6 . Из $\left(6.1_{5}\right)$ следует:

$$
\omega_{S}^{\prime}\left(x_{0}, x^{\prime}\right)=0, \quad \omega_{S}^{\prime}\left(x_{1}, x^{\prime}\right)=0 \quad \forall x^{\prime} \perp \Gamma .
$$

Из условий теоремы 6 вытекает, что $\forall x^{\prime} \in \Gamma$

$$
\begin{aligned}
& u_{0} A x^{\prime}+\sigma p_{1} x^{\prime}+\left(p_{0}, K x^{\prime}\right)+\left(Q x_{0}, x^{\prime}\right)=0 \\
& u_{1} A x^{\prime}-\sigma p_{0} x^{\prime}+\left(p_{1}, K x^{\prime}\right)+\left(Q x_{1}, x^{\prime}\right)=0 .
\end{aligned}
$$

Следовательно,

$$
\begin{aligned}
& u_{0} A+\sigma p_{1}+p_{0} K+Q x_{0} \in \Gamma, \\
& u_{1} A-\sigma p_{0}+p_{1} K+Q x_{1} \in \Gamma .
\end{aligned}
$$

Умножая левую часть первого включения на $x_{1}$ и пользуясь формулой (6.2), получаем:

$$
\frac{1}{2} \omega_{S}^{\prime}\left(x_{0}, x_{1}\right)=u_{0} A x_{1}+\sigma p_{1} x_{1}+p_{0} K x_{1}+Q x_{0} x_{1}
$$

HO

$$
\begin{aligned}
& u_{0} A x_{1}=-u_{0} u_{1}-u_{0} B^{*} p_{1} \\
& \sigma p_{1} x_{1}=p_{1} K x_{0}+p_{1} B u_{0}
\end{aligned}
$$

Следовательно,

$$
\frac{1}{2} \omega_{S}^{\prime}\left(x_{0}, x_{1}\right)=-u_{0} u_{1}+p_{1} K x_{0}+p_{0} K x_{1}+Q x_{0} x_{1}
$$

Отсюда согласно условиям 6 ) теоремы 6 следует: $\frac{1}{2} \omega_{S}^{\prime}\left(x_{0}, x_{1}\right)=0$. Но тогда из (6.4) следует, что

$$
\begin{aligned}
& u_{0} A+\sigma p_{1}+p_{0} K+Q x_{0}=\kappa_{0} x_{0} \\
& u_{1} A-\sigma p_{0}+p_{1} K+Q x_{1}=\kappa_{1} x_{1}
\end{aligned}
$$


Умножим первое равенство на $x_{0}$. Получим

$$
\omega_{S}\left(x_{0}\right)=u_{0} A x_{0}+\sigma p_{1} x_{0}+p_{0} K x_{0}+Q x_{0} x_{0}=\kappa_{0} x_{0}^{2},
$$

но

$$
\begin{gathered}
u_{0} A x_{0}=-u_{0}^{2}-u_{0} B^{*} p_{0}, \\
\sigma p_{1} x_{0}=\sigma p_{0} x_{1}=p_{0} K x_{0}+p_{0} B u_{0} .
\end{gathered}
$$

Следовательно,

$$
\omega_{S}\left(x_{0}\right)=-u_{0}^{2}+2 p_{0} K x_{0}+Q x_{0} x_{0} .
$$

Согласно условиям 6 ) теоремы 6 отсюда следует: $\omega_{S}\left(x_{0}\right)=\kappa x_{0}^{2}$. Следовательно, $\kappa_{0}=\kappa$. Аналогично доказывается, что $\kappa_{1}=\kappa$, тем самым необходимость доказана.

Докажем достаточность. Пусть набор $\sigma, \kappa ; x_{0}, x_{1} ; p_{0}, p_{1} ; u_{0}, u_{1}$ удовлетворяет всем условиям теоремы 7 . Выберем матрицу $S$ так, чтобы

$$
S x_{0}=p_{0}, \quad S x_{1}=p_{1} .
$$

В силу условия 3 ) теоремы такой выбор возможен. В остальном $S$ - произвольная симметрическая матрица. Мы покажем, что $S$ согласована с Г. Из условий теоремы легко следует:

$$
\begin{array}{ll}
U_{S} x_{0}=u_{0}, & U_{S} x_{1}=u_{1}, \\
R_{S} x_{0}=\sigma x_{1}, & R_{S} x_{1}=-\sigma x_{0} .
\end{array}
$$

Следовательно, выполнены условия $\left(6.1_{1}\right)-\left(6.1_{3}\right)$. Условия $\left(6.1_{4}\right),\left(6.1_{5}\right)$ легко вытекают из условия 6) теоремы. Мы не будем подробно останавливаться на этом, ибо подобные рассмотрения уже были, когда мы доказьвали необходимость. Достаточность, а вместе с ней и вся теорема, доказана.

Обратим внимание на то, что, как следует из доказательств теорем $4-7$, коль скоро пара $\Gamma, S$ согласована (слабо согласована), то и пара $\Gamma, S^{\prime}$ обладает тем же свойством для любой матрицы $S^{\prime}$, совпадающей на $\Gamma$ с $S$.

Условия 1 и 2 приобретают теперь следующую форму. Для любого набора, удовлетворяющего условиям одной из теорем $4-7$, должно иметь место неравенство $\kappa \geqslant 0$. Вероятно, есть возможность получить условия неотрицательности, в которых будут существенны некоторые свойства матрицы $S$ и вне $\Gamma$.

6.5. В этом пункте мы рассмотрим управляемую систему $V$ вида $\dot{x}=u$. В этом случае

$$
\begin{gathered}
U_{S}=R_{S}=-(A+S), \\
\omega_{S}(x)=-\left(U_{S} x\right)^{2}+Q x x .
\end{gathered}
$$

Матрицу $A$ в данном случае можно считать кососимметрической, что мы и будем делать. Матрицу $Q$ естественно предположить неотрицательно определенной. Тогда условия 1 и 2 можно не рассматривать для одномерных Г. Мы рассмотрим 
условие неотрицательности, опираюшееся на теорему 6. В нашем случае оно имеет следующий вид: для любых $\sigma, \kappa ; x_{0}, x_{1} ; p_{0}, p_{1} ; u_{0}, u_{1}$, удовлетворяющих условиям

$$
\begin{aligned}
& \sigma \neq 0, \\
& d\left(\operatorname{Lin}\left(x_{0}, x_{1}\right)\right)=2, \quad\left(x_{0}, x_{1}\right)=0, \\
& p_{1} x_{0}=p_{0} x_{1}, \\
& \sigma x_{1}=u_{0},-\sigma x_{0}=u_{1}, \\
& u_{0}=-\left(A x_{0}+p_{0}\right), u_{1}=-\left(A x_{1}+p_{1}\right), \\
& -u_{0}^{2}+\left(Q x_{0}, x_{0}\right)=\kappa x_{0}^{2},-u_{1}^{2}+\left(Q x_{1}, x_{1}\right)=\kappa x_{1}^{2}, \quad\left(Q x_{0}, x_{1}\right)=0,
\end{aligned}
$$

должно выполняться неравенство

$$
\kappa \geqslant 0
$$

Отметим, что из $\left(6.6_{2}\right)$ и $\left(6.6_{4}\right)$ следует $\left(u_{0}, u_{1}\right)=0$. Из условий $\left(6.6_{1}\right)-\left(6.6_{6}\right)$ легко исключить $u_{0}, u_{1}$ и выразить $\sigma$ через $x_{0}, x_{1}$. Из $\left(6.6_{4}\right),\left(6.6_{5}\right)$ имеем:

$$
\begin{aligned}
\sigma x_{1} & =-A x_{0}-p_{0}, \\
-\sigma x_{0} & =-A x_{1}-p_{1} .
\end{aligned}
$$

Умножая первое равенство на $x_{1}$, второе на $x_{0}$, вычитая одно из другого и учитывая (6.63), получаем:

$$
\sigma\left(x_{0}^{2}+x_{1}^{2}\right)=2 A x_{1} x_{0} .
$$

Условия (6.66) предстанут в виде:

$$
\begin{aligned}
-\sigma^{2} x_{1}^{2}+Q x_{0} x_{0} & =\kappa x_{0}^{2}, \\
-\sigma^{2} x_{0}^{2}+Q x_{1} x_{1} & =\kappa x_{1}^{2}, \\
Q x_{0} x_{1} & =0 .
\end{aligned}
$$

К этим условиям следует добавить еше условия $\left(6.6_{1}\right),\left(6.6_{2}\right)$. Введем обозначения:

$$
\begin{aligned}
\bar{x}_{0} & =\frac{x_{0}}{\left|x_{0}\right|}, & \bar{x}_{1} & =\frac{x_{1}}{\left|x_{1}\right|}, \\
\mu & =\frac{x_{0}^{2}}{x_{1}^{2}}, & a & =4\left(A \bar{x}, \bar{x}_{0}\right)^{2}, \\
q_{0} & =\left(Q \bar{x}_{0}, \bar{x}_{0}\right), & q_{1} & =\left(Q \bar{x}_{1}, \bar{x}_{1}\right) .
\end{aligned}
$$

В этих обозначениях условия (6.9) примут вид:

$$
\begin{gathered}
-\frac{a}{(1+\mu)^{2}}+q_{0}=\kappa, \\
-\frac{a}{(1+1 / \mu)^{2}}+q_{1}=\kappa, \\
a>0, \quad 0<\mu<+\infty .
\end{gathered}
$$


Выясним, когда условия (6.10) совместны, и найдем выражение для $\mu$. Имеем:

$$
q_{0}-q_{1}=a\left(\frac{1}{(1+\mu)^{2}}-\frac{1}{(1+1 / \mu)^{2}}\right)=a \frac{1-\mu^{2}}{(1+\mu)^{2}}=a \frac{1-\mu}{1+\mu}
$$

Отсюда

$$
\mu=\frac{a-\Delta q}{a+\Delta q}, \quad \Delta q=q_{0}-q_{1} .
$$

Следовательно, условия (6.10) совместны тогда и только тогда, когда

$$
a>|\Delta q| \text {. }
$$

Выясним условия, эквивалентные условию $\kappa \geqslant 0$. Оказывается, что эти условия можно сформулировать только через $a$ и $q$.

Действительно, пусть $\kappa \geqslant 0$. Тогда согласно (6.10) имеем:

$$
q_{0} \geqslant \frac{a}{(1+\mu)^{2}}, \quad q_{1} \geqslant \frac{a}{(1+1 / \mu)^{2}} .
$$

Отсюда

$$
\sqrt{q_{0}} \geqslant \frac{\sqrt{a}}{1+\mu}, \quad \sqrt{q_{1}} \geqslant \frac{\sqrt{a}}{1+1 / \mu} .
$$

Складывая, получаем: $\sqrt{q_{0}}+\sqrt{q_{1}} \geqslant \sqrt{a}$.

Пусть теперь $\kappa<0$. Тогда

$$
\sqrt{q_{0}}<\frac{a}{1+\mu}, \quad \sqrt{q_{1}}<\frac{a}{1+1 / \mu}
$$

откуда $\sqrt{q_{0}}+\sqrt{q_{1}}<\sqrt{a}$. Итак, условие, эквивалентное неравенству $\kappa \geqslant 0$, имеет вид:

$$
\sqrt{q_{0}}+\sqrt{q_{1}} \geqslant \sqrt{a}
$$

Заметим, что из неравенства $\sqrt{q_{0}}+\sqrt{q_{1}}<\sqrt{a}$ следует неравенство (6.12). Поэтому условие неотрицательности можно записать в виде:

$$
\begin{gathered}
\sqrt{q_{0}}+\sqrt{q_{1}} \geqslant \sqrt{a} \\
\forall \bar{x}_{0}, \bar{x}_{1}: \quad\left|\bar{x}_{0}\right|=\left|\bar{x}_{1}\right|=1,\left(\bar{x}_{0}, \bar{x}_{1}\right)=0, \quad\left(Q \bar{x}_{0}, \bar{x}_{1}\right)=0 .
\end{gathered}
$$

Это условие ранее не было известно. Такое условие для двумерного случая получил и обосновал А.В. Дмитрук [2]. Условие (6.14) можно трактовать как требование выполнения условия Дмитрука для любой плоскости.

Действительно, рассмотрим траектории, лежащие в плоскости Г. Тогда форму $\Phi$ можно записать в виде:

$$
\Phi=u^{2}+2(P A P x, u)+(P Q P x, x)
$$

где $P$ - ортогональный проектор на Г. Тогда $q_{0}$ и $q_{1}$ - собственные векторы оператора $P Q P$, а величина $\sqrt{a} / 2$ равна модулю множителя, соответствуюшего оператору $P A P$ при повороте на $\pi / 2$. Тогда (6.14) как раз совпадет с условием Дмитрука для траекторий плоскости Г. Условие Дмитрука можно получить и исходя из частотного критерия, однако оно не вытекает из последнего естественньм путем. 
§7. Некоторый класс квадратичных форм, приводящихся к формам с постоянными коэффициентами

Рассмотрим пару $\Phi, V$ вида

$$
\begin{gathered}
\Phi=a^{2}(t) u^{2}+2 m(t)(A x, u)+q^{2}(t)(Q x, x), \\
V: \quad \dot{x}=k(t) K x+b(t) B u .
\end{gathered}
$$

Соответствуюшая форма имеет вид $J=\int_{0}^{\infty} \Phi(x(t), u(t), t) d t$ и рассматривается на $L(V)$. Матрицы $A, Q, K, B$ постоянны. Коэффициенты $a(t), m(t), q(t), k(t), b(t)$ положительны на полуоси. Будем считать, что выполнены требования:

$$
\begin{aligned}
& m=a q ; \\
& \frac{q^{2}}{k}=\frac{m}{b} ; \\
& \frac{d}{d t}\left(\frac{m}{b}\right)=\lambda_{0} K \frac{m}{b}, \quad \lambda_{0}=\mathrm{const} ; \\
& \int_{0}^{\infty} k(t) d t=+\infty .
\end{aligned}
$$

7.1. В этом пункте мы покажем, что коль скоро выполнены $\left(7.2_{1}\right)-\left(7.2_{4}\right)$, то паpy $\Phi, V$ и форму $J$ можно привести заменой переменных к паре и форме с постоянньми коэффициентами. Положим $\tau(t)=\int_{0}^{t} k(t) d t$. Докажем, что

$$
e^{-\lambda_{0} \tau(t)}=\frac{m(0)}{b(0)} \cdot \frac{b(t)}{m(t)}
$$

Действительно, из (7.21 $)-\left(7.2_{3}\right)$ вытекает

$$
\frac{d}{d t}\left(\frac{m}{b} e^{-\lambda_{0} \tau}\right)=\left(\lambda_{0} k \frac{m}{b}-\lambda_{0} k \frac{m}{b}\right) e^{-\lambda_{0} \tau}=0,
$$

откуда следует (7.3).

Рассмотрим теперь замену: $(x, u, t) \rightarrow(\bar{x}, \bar{u}, \tau)$, где

$$
\begin{aligned}
\tau & =\tau(t), \\
x & =e^{-\frac{\lambda_{0}}{2} \tau} \bar{x} \\
u & =\frac{q}{a} e^{-\frac{\lambda_{0}}{2} \tau} \bar{u}
\end{aligned}
$$

Тогда

$$
\Phi(x, u, t)=q^{2}(t) e^{-\lambda_{0} \tau(t)}\left(\bar{u}^{2}+2 A \bar{x} \bar{u}+Q \bar{x} \bar{x}\right) .
$$

Полагая $\bar{\Phi}(\bar{x}, \bar{u})=\bar{u}^{2}+2 A \bar{x} \bar{u}+Q \bar{x} \bar{x}$, получаем:

$$
\Phi(x, u, t)=q^{2}(t) e^{-\lambda_{0} \tau(t)} \bar{\Phi}(\bar{x}, \bar{u}) .
$$


Согласно (7.3) получаем отсюда:

$$
\Phi(x, u, t) d t=\frac{m(0)}{b(0)} \bar{\Phi}(\bar{x}, \bar{u}) d \tau .
$$

Подставляя далее в $V$ вместо $x, u, t$ их выражения через $\bar{x}, \bar{u}, \tau$, получаем:

$$
k \frac{d}{d \tau} e^{-\frac{\lambda_{0}}{2} \tau} \bar{x}=k e^{-\frac{\lambda_{0}}{2} \tau} K \bar{x}+\frac{b q}{a} e^{-\frac{\lambda_{0}}{2} \tau} B \bar{u} .
$$

Сокращая на экспоненту и $k$, получаем:

$$
\frac{d \bar{x}}{d \tau}=\left(\frac{\lambda_{0}}{2} I+K\right) \bar{x}+\frac{b q}{k a} B \bar{u}
$$

Но из $\left(7.2_{1}\right),\left(7.2_{2}\right)$ следует $\frac{b q}{k a}=1$. Таким образом, управляемая система $V$ переходит в управляемую систему $\bar{V}$ :

$$
\frac{d \bar{x}}{d \tau}=\left(\frac{\lambda_{0}}{2} I+K\right) \bar{x}+B \bar{u}
$$

При этом форма $J$ переходит, с точностью до постоянного множителя, в форму $\bar{J}=\int_{0}^{\infty} \bar{\Phi} d \tau$. Итак, доказано, что если коэффициенты $a, m, q, k, b$ удовлетворяют $\left(7.2_{1}\right)-\left(7.2_{4}\right)$, то заменой (7.4) пара $\Phi, V$ переходит в пару $\bar{\Phi}, \bar{V}$ с постоянными коэффициентами, а форма $J$ в $\bar{J}$. Очевидно, $L(V)$ переходит в $L(\bar{V})$. Поэтому условие неотрицательности $\bar{J}$ на $L(\bar{V})$ является условием неотрицательности $J$ на $L(V)$.

7.2. Пусть пара $\Phi, V$ имеет вид (7.1). Полагая $v=b(t) u$, получаем новую пару $\Phi_{1}, V_{1}$, где $\Phi_{1}$ и правая часть $V_{1}$ зависят от $x, v, t$ так же, как в (7.1), с коэффициентами

$$
\frac{a^{2}}{b^{2}}, \frac{m}{b}, q^{2}, k, 1
$$

Нетрудно проверить, что если старые коэффициенты $a^{2}, m, q^{2}, k, b$ удовлетворяли условиям $\left(7.2_{1}\right)-\left(7.2_{4}\right)$, то и новые коэффициенты удовлетворяют этим условиям. Поэтому можно ограничиться случаем, когда $b=1$. Ниже мы будем считать это равенство выполненным.

Пусть пара $\Phi, V$ имеет вид:

$$
\begin{gathered}
\Phi=z(t)\left(\frac{1}{\rho(t)} u^{2}+2 A x u+\rho(t) Q x x\right) \\
V: \dot{x}=\rho K x+B u
\end{gathered}
$$

где $\rho(t), z(t)>0 ; \frac{d z}{d t}=\lambda \rho z ; \lambda=\mathrm{const} ; \int_{0}^{\infty} \rho d t=+\infty$.

Мы докажем два предложения. 
ПРЕДЛОЖЕНИЕ 3. Пусть пара $\Phi, V$ имеет вид (7.8). Тогда коэффициентьи $a, m, q, k, b$, əдe

$$
a=\sqrt{\frac{z}{\rho}}, \quad m=z, \quad q=\sqrt{z \rho}, \quad k=\rho, \quad b=1
$$

удовлетворяют условиям $\left(7.2_{1}\right)-\left(7.2_{4}\right)$, причем $\lambda_{0}=\lambda$.

Доказательство представляет собой простую проверку этих условий.

ПРЕДЛОЖЕНИЕ 4. Пусть пара $\Phi, V$ вида (7.1) такова, что $a, m, q, k, b$ удовлетворяют $\left(7.2_{1}\right)-\left(7.2_{4}\right)$, причем $b=1$. Тогда пару $\Phi, V$ можсно записать в виде (7.8).

ДоКАЗАТЕЛЬСТво. Положим $z(t)=m(t), \rho(t)=k(t)$. Тогда

$$
\Phi(x, u, t)=z(t)\left(\frac{a^{2}}{m} u^{2}+2 A x u+\frac{q^{2}}{m} Q x x\right) .
$$

Так как $b=1$, то из $\left(7.2_{1}\right)-\left(7.2_{3}\right)$ следует:

$$
\frac{a^{2}}{m}=\frac{1}{k}, \quad \frac{q^{2}}{m}=k, \quad \frac{d m}{d t}=\lambda_{0} k m .
$$

Тем самым предложение доказано.

Форма записи (7.8) весьма проста. Вместо четырех коэффициентов $a, m, q, k$ $(b=1$ !) она оперирует двумя: $z$ и $\rho$. Если $\rho=1$, то к классу (7.8) относится пара:

$$
\begin{gathered}
\Phi=e^{\lambda t}\left(u^{2}+2 A x u+Q x x\right), \\
V: \dot{x}=K x+B u
\end{gathered}
$$

при любом $\lambda$. Согласно п. 7.1 любая такая пара приводится к паре $\bar{\Phi}, \bar{V}$ с постоянньпи коэффициентами, где

$$
\begin{gathered}
\bar{\Phi}=\bar{u}^{2}+2 A \bar{x} \bar{u}+Q \bar{x} \bar{x} \\
\bar{V}: \frac{d \bar{x}}{d \tau}=\left(\frac{\lambda}{2} I+K\right) \bar{x}+B \bar{u}
\end{gathered}
$$

7.3. В этом пункте мы рассмотрим форму:

$$
\int_{0}^{1}\left(\theta^{\alpha} u^{2}+2 \theta^{\beta}(A x, u)+\theta^{\gamma}(Q x, x)\right) d \theta
$$

где

$$
\frac{d x}{d \theta}=\theta^{\mu} K x+B u, \quad x(1)=0
$$

и $x(\theta)=0, u(\theta)=0$ в некоторой окрестности нуля. Формы такого вида рассматривал В.А. Арутюнов [3] в связи с нарушением усиленного условия Лежандра. 
Мы выясним, при каких $\alpha, \beta, \gamma, \mu$ форма (7.9) и управляемая система (7.10) могут быть приведены к форме и управляемой системе с постоянными коэффициентами на полуоси. Положим $\kappa(t)=1+t$ и рассмотрим замену $\theta=1 / \kappa$. Тогда подьнтегральное выражение в (7.9) примет вид:

$$
\Phi(x, u, t) d t=\left(\frac{1}{\kappa^{\alpha+2}} u^{2}+2 \frac{1}{\kappa^{\beta+2}} A x u+\frac{1}{\kappa^{\gamma+2}} Q x x\right) d t,
$$

а управляемая система (7.10) - вид:

$$
V: \frac{d x}{d t}=-\frac{1}{\kappa^{\mu+2}} K x-\frac{1}{\kappa^{2}} B u .
$$

Выясним, когда для коэффициентов этой системы выполнены условия $\left(7.2_{1}\right)-\left(7.2_{4}\right)$. Полагая $v=u / \kappa^{2}$, переходим к паре $\Phi_{1}, V_{1}$ :

$$
\begin{gathered}
\Phi_{1}(x, v, t)=\kappa^{-\beta}\left(\kappa^{\beta-\alpha+2} v^{2}+2 A x v+\kappa^{\beta-\sigma-2} Q x x\right), \\
V_{1}: \frac{d x}{d t}=-\frac{1}{\kappa^{\mu+2}} K x-B v
\end{gathered}
$$

Из предложений 3 и 4 следует, что

$$
\alpha+\gamma=2 \beta, \quad \beta-\gamma=-\mu, \quad \mu+2 \leqslant 1
$$

Если $\beta \neq 0$, то $\frac{d}{d t}\left(\kappa^{-\beta}\right)=-\beta \kappa^{-\beta-1}$, откуда

$$
\lambda=-\beta, \quad \rho=\kappa^{-1}, \quad \mu=-1 .
$$

Если $\beta=0$, то

$$
\lambda=0, \quad \gamma=-\alpha=\mu, \quad \mu \leqslant-1, \quad \rho=\kappa^{-\mu-2}, \quad z=1 .
$$

При этом пара $\bar{\Phi}_{1}, \bar{V}_{1}$ имеет вид:

$$
\begin{gathered}
\bar{\Phi}_{1}=\bar{v}^{2}+2 A \bar{x} \bar{v}+Q \bar{x} \bar{x} \\
\bar{V}_{1}: \frac{d \bar{x}}{d \tau}=\left(-\frac{\beta}{2} I-K\right) \bar{x}-B \bar{v}
\end{gathered}
$$

а квадратичная форма $\bar{J}_{\text {имеет вид }}$

$$
\bar{J}=\int_{0}^{\infty} \bar{\Phi}_{1}(\bar{x}, \bar{v}) d \tau
$$

\section{Список литературы}

1. Барабанов Н.Е., Гелиг А.Х., Леонов Г.А., Лихтарников А. Л., Матвеев А.С., Смирнова В.Б., Фрадков А. Л. Частотная теорема (лемма Якубовича-Калмана) в теории управления // Автоматика и телемеханика. 1996. № 10. С. 3-40.

2. Дмитрук A. В. Критерий неотрицательности вырожденной квадратичной формы с двумерным управлением // Итоги науки и техн. Соврем. матем. и ее прилож. (в печати).

3. Арутюнов A. В. Условия экстремума. Анормальные и вырожденные задачи. М.: Факториал, 1997.

Институт химической физики РАН, г. Москва

Поступила в редакцию

19.09.2001 